\title{
Prevalência da infecção latente tuberculosa em comunicantes de portadores de tuberculose pulmonar
}

\author{
Prevalence of latent tuberculous infection in communicants of patients with pulmonary \\ tuberculosis
}

Prevalencia de la infección latente tuberculosa en comunicantes de portadores de tuberculosis pulmonar

Adilson Mendes de Figueiredo Júnior ${ }^{1 *}$, Antônia Margareth Moita Sá1.

\begin{abstract}
RESUMO
Objetivo: Identificar a prevalência da Infecção Latente Tuberculosa em comunicantes de portadores de Tuberculose pulmonar. Métodos: Estudo observacional, retrospectivo, realizado em um centro saúde escola da cidade de Belém/PA/Brasil, amostra de conveniência de 1.000 prontuários de comunicantes de portadores de tuberculose pulmonar. A coleta de dados foi realizada com um formulário e os dados analisados no software EpiInfo 3.5. Resultados: Detectou-se uma prevalência de Infecção Latente Tuberculosa (ILTB) de 50,7\%, com PT+ mais frequente entre comunicantes do sexo masculino $(51,80 \%)$ e comunicantes na faixa etária de 31 a 40 anos. O parentesco com maior frequência de Prova Tuberculínica (PT)+ (64,9\%) foi o "Pai/Mãe". A maior frequência de $\mathrm{PT}+$ relaciona-se aos que possuem casos índices, moradores de ruas estreitas, com residências até 3 cômodos e 3 janelas. Conclusão: A prevalência de infectados encontrada foi maior que a prevalência nacional de infectados.
\end{abstract}

Palavras-chave: Tuberculose, Tuberculose Latente, Saúde Pública.

\section{ABSTRACT}

Objective: To identify the prevalence of Latent Tuberculosis Infection in communicants of patients with pulmonary tuberculosis. Materials and Methods: Observational, retrospective study performed at a health school in the city of Belem / PA / Brazil, a convenience sample of 1,000 patient records of patients with pulmonary tuberculosis. Data collection was performed with a form and data analyzed in Epi-Info 3.5 software. Results: A prevalence of Infection Latent Tuberculous (ILTB) of $50.7 \%$ was found, with more frequent Tuberculin Test (TT)+ between male communicants $(51.80 \%)$ and communicants in the age range from 31 to 40 years. The most frequent relationship of PT + (64.9\%) was the "Father / Mother". The higher frequency of PT + is related to cases with index cases, residents of narrow streets, with residences up to 3 rooms and 3 windows. Conclusion: The prevalence of infected individuals was higher than the national prevalence of infected individuals.

Keywords: Tuberculosis, Latent Tuberculosis, Public health.

\section{RESUMEN}

Objetivo: Identificar la prevalencia de la Infección latente Tuberculosa en comunicantes de portadores de Tuberculosis pulmonar. Materiales y Métodos: Estudio observacional, retrospectivo, realizado en un centro salud escuela de la ciudad de Belém / PA / Brasil, muestra de conveniencia de 1.000 prontuarios de comunicantes de portadores de tuberculosis pulmonar. La recolección de datos se realizó con un formulario y los datos analizados en el software Epi-Info 3.5. Resultados: Se detectó una prevalencia de Infección latente tuberculosa (ILTB) de 50,7\%, con Prueba de tuberculina (PT)+ más frecuente entre comunicantes del sexo masculino $(51,80 \%)$ y comunicantes en el grupo de edad de 31 a 40 años. El parentesco con mayor frecuencia de PT + $(64,9 \%)$ fue el "Padre / Madre". La mayor frecuencia de PT + se relaciona con los que tienen casos índices, habitantes de calles estrechas, con residencias hasta 3 habitaciones y 3 ventanas. Conclusión: La prevalencia de infectados encontrados fue mayor que la prevalencia nacional de infectados.

Palabras clave: Tuberculosis, Tuberculosis Latente, Salud pública.

${ }^{1}$ Universidade do Estado do Pará (UEPA), Belém-PA. *E-mail: adilsonmdfi@hotmail.com 


\section{INTRODUÇÃO}

A Infecção Latente Tuberculosa (ILTB) é a infecção que antecede o aparecimento da Tuberculose ativa (TB), ou seja, constitui-se do período entre a entrada do M. tuberculosis no organismo e o aparecimento da doença. A detecção da ILTB para a Organização Mundial da Saúde (OMS) significa uma estratégia para o controle da tuberculose no mundo, pois a detecção da infecção latente permite o início do tratamento medicamentoso evitando a progressão para doença ativa (BRASIL, 2011).

A disposição à ILTB é praticamente universal. A maioria das pessoas não adoece após a infecção, o que leva as mesmas, a desenvolverem imunidade parcial à doença. Cerca de $5 \%$ das pessoas infectadas não conseguem evitar o crescimento bacilar e acabam adoecendo, enquanto outros $5 \%$ bloqueiam a infecção nessa fase, mas acabam adoecendo posteriormente devido à reativação bacilar ou por serem expostos a novas fontes de infecção. O adoecimento ocorre em sua maioria nos dois primeiros anos após a infecção, mas o período de incubação pode se estender por anos ou até décadas (BRASIL, 2011a).

No Brasil, cerca de 57 milhões de pessoas estão infectadas pelo bacilo causador da tuberculose, com maior incidência nas grandes cidades, e entre as populações vulneráveis o risco aumenta entre os comunicantes dos portadores de TB, na população carcerária, com taxas 25 vezes maiores que a população geral e nos portadores do HIV/Aids, com taxas 30 vezes maiores; dessa maneira, é possível observar a ligação direta da infecção latente a fatores sociais, necessitando assim, de uma maior atenção das ações do governo para a redução da vulnerabilidade em saúde da população (PILLER RVB, 2012).

O comunicante é todo indivíduo que convive em um mesmo ambiente que o caso índice, que por sua vez é todo paciente com TB pulmonar ativa apresentando baciloscopia positiva. O convívio pode ser tanto diário, dividindo uma mesma moradia, como eventual em ambientes de trabalho e escolar, visto que a avalição de cada grau de exposição deve levar em consideração a forma da doença, o ambiente e o tempo de exposição (contínuo ou descontínuo) (LAPA e SILVA JR, 2012).

O controle dos comunicantes dos casos de Tuberculose é considerado um mecanismo de extrema importância para a prevenção do adoecimento futuro. Comunicantes que deixam de ser acompanhados e/ou avaliados representam um fator importante para a manutenção da Tuberculose, pois ficam mais suscetíveis a desenvolverem futuramente a doença ativa. Dessa maneira, a transmissão dos bacilos para novos indivíduos torna-se mais frequente e pode futuramente incrementar as taxas de incidência da TB (ANDRADE HS et al, 2016).

O Brasil faz parte dos 22 países prioritários pela OMS para combate à Tuberculose, que concentram cerca de $80 \%$ da carga mundial de TB. Em 2015, 63.189 casos novos foram notificados no Brasil, correspondendo a um coeficiente de incidência de 30.9/100.000 habitantes e uma taxa de mortalidade de 2,2/100.000 habitantes. O Brasil ocupa a 18ํㅜ posição em relação à carga de Tuberculose (BRASIL, 2016).

Os casos de Tuberculose estão distribuídos em todo território nacional, principalmente em 181 municípios que são prioritários no combate a doença, e juntos correspondem a $70 \%$ do total de casos no Brasil; o grupo na faixa etária entre os 15 e 59 anos é o mais atingido pela Tuberculose e dentre as populações mais vulneráveis, a incidência em negros é 2 vezes maior que a média nacional da população geral, já na população indígena essa incidência é 4 vezes maior (BRASIL, 2014). Este estudo visa contribuir para incrementar a prevenção da Tuberculose entre os comunicantes de portadores da doença, reduzindo a carga de infectados, e diminuindo as chances de adoecimentos futuros, ao analisar, sobretudo, as características clínico-epidemiológicas e sociodemográficas que podem determinar ou não a presença da Infecção Latente Tuberculosa. O estudo teve como objetivo identificar a prevalência da Infecção Latente Tuberculosa em comunicantes de portadores de Tuberculose pulmonar.

\section{MÉTODOS}

É um estudo observacional, retrospectivo, do tipo ecológico realizado em condições de rotina. O estudo foi realizado no Centro de Saúde Escola do Marco (CSE-Marco). O Centro é uma instituição pública, de gestão estadual, coordenada pela Universidade do Estado do Pará (UEPA), e atende usuários provenientes do município de Belém e região metropolitana, bem como de outros municípios do Pará.

A população do estudo foi composta por comunicantes de portadores de tuberculose, submetidos a PT no CSE-Marco no período de 2009 a 2013, o que representa uma população média de 1500 indivíduos. Os dados foram obtidos através de consultas aos prontuários dos usuários.

A amostra foi constituída por 1000 prontuários de comunicantes, o que representou cerca de $65 \%$ da população média. Optou-se em uma amostra de conveniência com 200 prontuários anuais, nos períodos de 2009 a 2013. A amostra foi composta por prontuários de usuários de ambos os sexos em qualquer faixa etária, comunicantes de portadores de tuberculose, que no período de 2009 a 2013, foram atendidos no CSE-Marco, para se submeterem a PT. Utilizou-se um formulário considerando como variáveis independentes: Sexo, faixa etária, positividade da baciloscopia dos casos índices, relação de parentesco, a intensidade do convívio com caso índice, o tempo decorrido entre o surgimento de sintomas e o início do tratamento nos casos índices, 
número de doses da BCG e as condições de moradia; e como variável dependente: resultado da prova tuberculínica, considerado positivo quando for igual ou maior que $05 \mathrm{~mm}$. Os dados foram coletados em condições de rotina, através do formulário adaptado do prontuário para atendimento de contatos de portadores de tuberculose do CSE-Marco. Aos resultados obtidos aplicou-se o teste qui-quadrado para identificar as variáveis preditoras que apresentavam associação significativa com a variável desfecho, respeitando os resultados de $p$-valor limítrofe de 0,05 .

Aos dados estatisticamente significativos que foram encontrados no estudo, aplicou-se a regressão logística multivariada identificando as razões das chances. A Regressão Logística permitiu a predição de valores entre uma variável dependente e variáveis independentes para determinar fatores que caracterizam os grupos com maior probabilidade de chances para a Infecção Latente Tuberculosa. Os dados coletados foram registrados em um banco de dados confeccionado com o software Epi Info® versão 3.5.1. Em seguida, utilizou-se o programa BioEstat 5.0 para tratamento, análise e para verificar a associação entre as variáveis do estudo. A pesquisa foi aprovada pelo Comitê de Ética em Pesquisa da UEPA, CAAE no 30932814.3.0000.5170.

A Regressão Logística múltipla foi aplicada sobre as variáveis independentes que, através do cálculo do quiquadrado e dos valores de $p$-valor $(<0,05)$, apresentaram associação estatística significativa. $O$ cálculo estatístico da OR diferenciou as variáveis de pouca associação das de grande associação. Todas as variáveis com razões de chances < 1,0 são consideradas de baixa relação de dependência. Já as variáveis com razões de chances > 1,0 são consideradas de forte relação de dependência, ou seja, estão ligadas diretamente com a possibilidade da infecção pela ILTB. Pode-se observar ainda a razão de chances (Odds Ratio) com seu intervalo de confiança $\alpha=0.05$ (L.I - Limite Inferior e L.S - Limite Superior).

\section{RESULTADOS}

Trabalhou-se com uma amostra de 1.000 prontuários de comunicantes de portadores de TB ativa, que realizaram a Prova Tuberculínica (PT) no CSE-MARCO entre os anos de 2009 a 2013. Destes, 506 apresentaram PT+ constituindo rendimento de $50,7 \%$, o que representa uma frequência maior que a prevalência média de infectados pelo M. tuberculosis no Brasil, que é estimada pelo Ministério da Saúde em 30\% (OLIVEIRA et al, 2008). Na Tabela 1 pode-se observar que a maioria dos pacientes submetidos à Prova Tuberculínica foi do sexo feminino. A PT+ foi mais frequente entre os homens correspondendo a $51,80 \%$. Pode-se observar também que as variáveis "sexo" e "Rendimento da PT" não se apresentaram associadas estatisticamente ( $p$-valor $=0.563)$.

Tabela 1 - Rendimento da Prova Tuberculínica em comunicantes de Tuberculose pulmonar segundo o sexo, faixa etária e o parentesco.

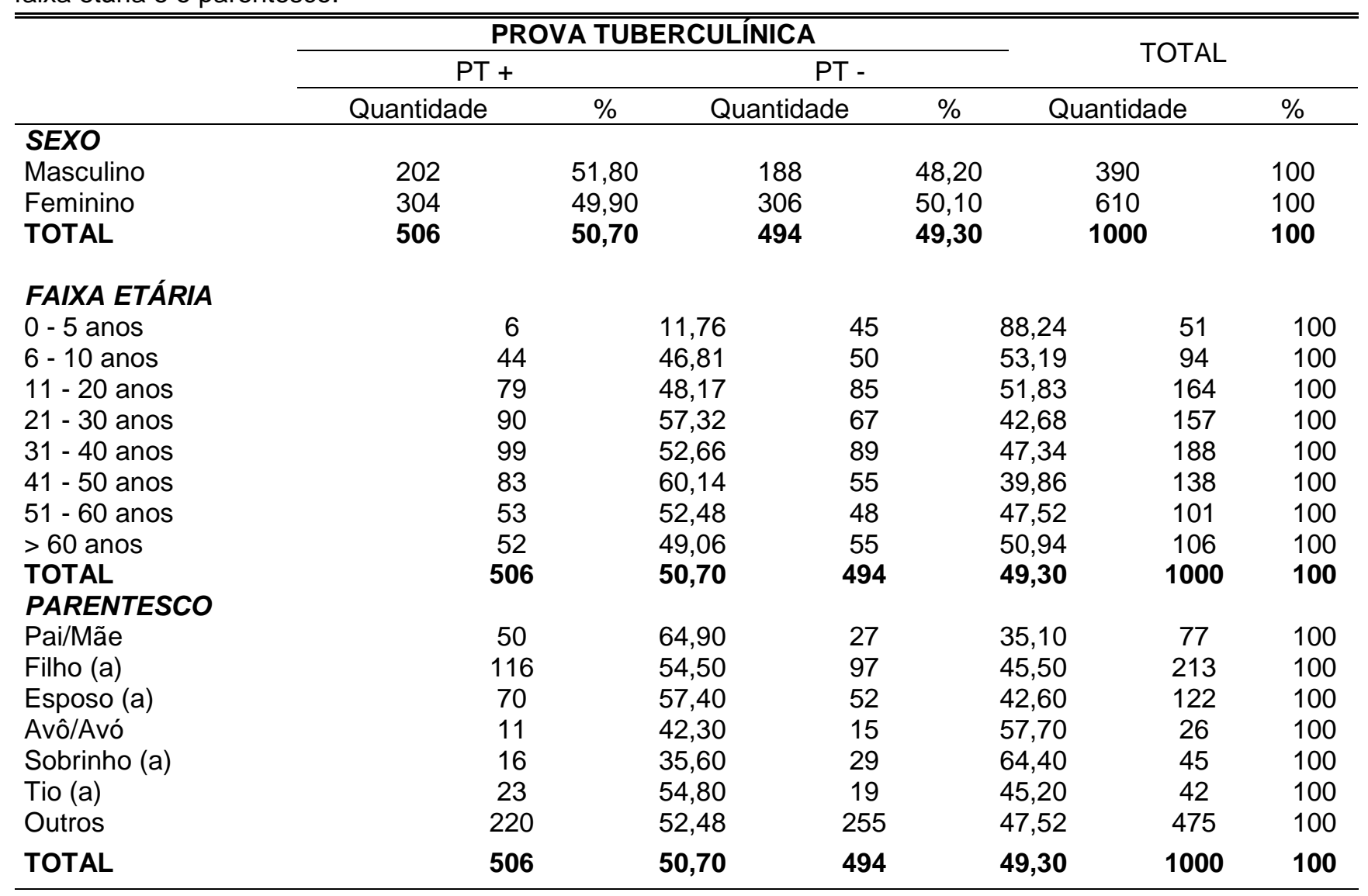

*Fonte: Protocolo de pesquisa, 2015.

†PT +: Prova Tuberculínica positiva; PT -: Prova Tuberculínica negativa.

$\ddagger$ Sexo. $P=0,563 . X^{2}=0,335$.

§Faixa Etária. $P=0,020 . X^{2}=40,131$.

\| Parentesco. $P=, 006 . X^{2}=18,245$. 
Em relação à faixa etária observa-se que dos 1.000 contatos submetidos à Prova Tuberculínica 188 encontravam-se na faixa etária de 31 a 40 anos representando a maior frequência. A faixa etária de 41 a 50 anos concentrou o maior número de PT + com $60,14 \%$, enquanto que a faixa etária de 0 a 5 anos concentrou o maior número de PT- com $88,24 \%$. Pode-se observar também que as variáveis "faixa etária" e "Rendimento da PT" se apresentaram associadas estatisticamente ( $p$-valor $=0,020)$.

Quando se relaciona o parentesco com a prova tuberculínica é possível observar, ainda de acordo com a Tabela 1, que o grupo "Pai/Mãe" apresentou a maior frequência de PT+ com 64,90\% seguido do grupo "Esposo(a)" com frequência de PT+ de $57,40 \%$. O grupo que apresentou a menor frequência de PT+ foi "sobrinho (a)" com $35,60 \%$. As variáveis "parentesco" e "Rendimento da PT" se apresentaram associadas estatisticamente $(\mathrm{p}$-valor $=0,006)$.

Na Tabela 2 observa-se que dos 1.000 pacientes submetidos à Prova Tuberculínica 154 não eram vacinados com a BCG, com frequência de PT+ em 63,0\%. Porém, o grupo de vacinados com 2 doses apresentou a maior frequência de PT+ com 68,2\%. Pode-se observar também que as variáveis "Vacinado com BCG" e "Rendimento da PT" se apresentaram associadas estatisticamente $(p$-valor=0.010). Em relação ao tipo de convívio, o mais frequente entre contatos e portadores de TB ativa é o contínuo $(n=915)$, além de representar a maior frequência de PT+ (52,80\%). Pode-se observar também que as variáveis "Tipo de convívio" e "Rendimento da PT" se apresentaram associadas estatisticamente ( $p$-valor $=0,050)$.

Tabela 2 - Rendimento da Prova Tuberculínica em comunicantes de Tuberculose pulmonar segundo vacinação com a BCG e tipo de convívio.

\begin{tabular}{|c|c|c|c|c|c|c|}
\hline & \multicolumn{4}{|c|}{ PROVA TUBERCULIINICA } & \multirow{2}{*}{\multicolumn{2}{|c|}{ TOTAL }} \\
\hline & \multicolumn{2}{|c|}{$\mathrm{PT}+$} & \multicolumn{2}{|l|}{ PT - } & & \\
\hline & Quantidade & $\%$ & Quantidade & $\%$ & Quantidade & $\%$ \\
\hline \multicolumn{7}{|l|}{ BCG } \\
\hline Não vacinado & 97 & 63,00 & 57 & 37,00 & 154 & 100 \\
\hline 1 Dose & 378 & 47,30 & 422 & 52,80 & 800 & 100 \\
\hline 2 Doses & 31 & 68,20 & 15 & 31,80 & 44 & 100 \\
\hline TOTAL & 506 & 50,70 & 494 & 49,30 & 1000 & 100 \\
\hline \multicolumn{7}{|l|}{ TIPO DE CONVÍVIO } \\
\hline Descontínuo (eventual) & 23 & 27,40 & 61 & 72,60 & 84 & 100 \\
\hline $\begin{array}{l}\text { Contínuo (diário) } \\
\text { TOTAL }\end{array}$ & $\begin{array}{l}483 \\
506\end{array}$ & $\begin{array}{l}52,80 \\
50,70\end{array}$ & $\begin{array}{l}432 \\
494\end{array}$ & $\begin{array}{l}47,20 \\
49,30\end{array}$ & $\begin{array}{c}915 \\
1000\end{array}$ & $\begin{array}{l}100 \\
100\end{array}$ \\
\hline
\end{tabular}

${ }^{\star}$ Fonte: Protocolo de pesquisa, 2015.

†PT +: Prova Tuberculínica positiva; PT -: Prova Tuberculínica negativa.

BCG. $P=0,010 \cdot X^{2}=18,486$.

$\S$ Tipo de Convívio. $P=0,050 . X^{2}=19,867$.

$\mathrm{Na}$ Tabela 3 verifica-se que dos 1.000 indivíduos submetidos a PT, 211 possuíam caso índice com baciloscopia positiva de três cruzes $(+++)$ e apresentaram maior frequência de positividade $(76,30 \%)$. As variáveis "Baciloscopia inicial" e "Rendimento da PT" se apresentaram associadas estatisticamente ( $p$-valor = 0 , 001).

Tabela 3 - Rendimento da Prova Tuberculínica em comunicantes de Tuberculose pulmonar segundo a baciloscopia inicial do caso índice e o tempo entre os primeiros sintomas e início do tratamento.

\begin{tabular}{|c|c|c|c|c|c|c|}
\hline & \multicolumn{4}{|c|}{ PROVA TUBERCULÍNICA } & \multirow{2}{*}{\multicolumn{2}{|c|}{ TOTAL }} \\
\hline & \multicolumn{2}{|c|}{$\mathrm{PT}+$} & \multicolumn{2}{|l|}{ PT - } & & \\
\hline & Quantidade & $\%$ & Quantidade & $\%$ & Quantidade & $\%$ \\
\hline \multicolumn{7}{|c|}{ BACILOSCOPIA INICIAL } \\
\hline+ & 146 & 33,00 & 296 & 67.00 & 442 & 100 \\
\hline ++ & 199 & 57,50 & 148 & 42,50 & 347 & 100 \\
\hline+++ & 161 & 76,30 & 50 & 23,70 & 211 & 100 \\
\hline TOTAL & 506 & 50,70 & 494 & 49,30 & 1000 & 100 \\
\hline \multicolumn{7}{|c|}{$\begin{array}{l}\text { INCIO DOS SINTOMAS X INÍCIO DO } \\
\text { TRATAMENTO }\end{array}$} \\
\hline Menos de 1 mês & 57 & 25,00 & 172 & 75.00 & 229 & 100 \\
\hline 2 a 3 meses & 109 & 36,90 & 186 & 63,10 & 295 & 100 \\
\hline 4 a 5 meses & 155 & 73,80 & 55 & 26,20 & 210 & 100 \\
\hline Mais de 5 meses & 185 & 69,50 & 81 & 30,50 & 266 & 100 \\
\hline TOTAL & 506 & 50,70 & 494 & 49,30 & 1000 & 100 \\
\hline
\end{tabular}

${ }^{*}$ Fonte: Protocolo de pesquisa, fevereiro/2015.

†PT +: Prova Tuberculínica positiva; PT -: Prova Tuberculínica negativa.

$\ddagger$ Baciloscopia Inicial. $P=0,001 . X^{2}=116,964$. 
$\S$ Tempo Sintomas $x$ Tratamento. $P=0,001$.

$X^{2}=165,238$.

Em relação ao tempo entre o início dos primeiros sintomas da doença e o início do tratamento, dos 1.000 indivíduos submetidos a PT, 210 possuíam caso índice que levaram de 04 a 05 meses entre os primeiros sintomas da doença e o início do tratamento, e apresentaram maior frequência de positividade no teste $(73,80 \%)$. Pode-se observar também que as variáveis "Tempo entre os primeiros sintomas e o início do tratamento" e "Rendimento da PT" se apresentaram associadas estatisticamente ( $p$-valor $=0,001)$.

Na Tabela 4 a maior frequência de PT+ $(65,10 \%)$ relaciona-se aos indivíduos (comunicantes) que possuem como casos índices, moradores de ruas estreitas. As variáveis "Localização" e "Rendimento da PT" se apresentaram associadas estatisticamente ( $p$-valor $=0$, 002). Dos 1.000 indivíduos submetidos a PT, 319 possuíam como casos índices, moradores de casas com 1 a 3 cômodos, e apresentaram a maior frequência de PT+ (73,04\%). Observou-se que as variáveis "Número de cômodos" e "Rendimento da PT" se apresentaram associadas estatisticamente ( $\mathrm{p}$-valor $=0,001)$.

Outra variável estudada foi o número de janelas da residência do caso índice. A maior frequência de PT+ $(65,51 \%)$ relaciona-se aos indivíduos (comunicantes) que possuem como casos índices, moradores de casas com 1 a 3 janelas. As variáveis "Número de janelas" e "Rendimento da PT" se apresentaram associadas estatisticamente $(p$-valor $=0,001)$.

Tabela 4 - Rendimento da Prova Tuberculínica em comunicantes de Tuberculose pulmonar segundo a localização do caso índice e o número de cômodos e janelas da residência do caso índice.

\begin{tabular}{|c|c|c|c|c|c|c|}
\hline & \multicolumn{4}{|c|}{ PROVA TUBERCULÍNICA } & \multirow{2}{*}{\multicolumn{2}{|c|}{ TOTAL }} \\
\hline & \multicolumn{2}{|c|}{$\mathrm{PT}+$} & \multicolumn{2}{|l|}{ PT - } & & \\
\hline & Quantidade & $\%$ & Quantidade & $\%$ & Quantidade & $\%$ \\
\hline \multicolumn{7}{|c|}{ LOCALIZAÇÃO DO CASO ÍNDICE } \\
\hline Passagem & 161 & 50,50 & 159 & 49,50 & 320 & 100 \\
\hline Rua estreita & 84 & 65,10 & 45 & 34,90 & 129 & 100 \\
\hline Rua larga & 223 & 46,50 & 257 & 53,50 & 480 & 100 \\
\hline Vila & 38 & 53,50 & 33 & 46,50 & 71 & 100 \\
\hline \multicolumn{7}{|c|}{ No DE CÔMODOS DO CASO ÍNDICE } \\
\hline 1 a 3 cômodos & 233 & 73,04 & 86 & 26,96 & 319 & 100 \\
\hline 4 a 5 cômodos & 139 & 42,00 & 192 & 58,00 & 331 & 100 \\
\hline > 5 cômodos & 134 & 38,30 & 216 & 61,70 & 350 & 100 \\
\hline TOTAL & 506 & 50,70 & 494 & 49,30 & 1000 & 100 \\
\hline \multicolumn{7}{|c|}{ No DE JANELAS DO CASO ÍNDICE } \\
\hline 1 a 3 janelas & 323 & 65,51 & 170 & 34,49 & 493 & 100 \\
\hline 4 a 5 janelas & 92 & 39,70 & 140 & 60,30 & 232 & 100 \\
\hline$>5$ janelas & 91 & 33,10 & 184 & 66,90 & 275 & 100 \\
\hline TOTAL & 506 & 50,70 & 494 & 49,30 & 1000 & 100 \\
\hline
\end{tabular}

* Fonte: Protocolo de pesquisa, fevereiro/2015.

†PT +: Prova Tuberculínica positiva; PT -: Prova Tuberculínica negativa.

łLocalização do Caso índice. $P=0,002$.

$X^{2}=14,413$.

§Número de Cômodos. $P=0,001 . X^{2}=102,309$.

||Número de Janelas. $P=0,001 . X^{2}=89,905$.

Tabela 5 - Regressão logística das variáveis estatisticamente independentes.

\begin{tabular}{lcccccc}
\hline \multirow{2}{*}{ VARIÁVEIS } & \multicolumn{3}{c}{} & \multicolumn{2}{c}{ IC 95\% } \\
\cline { 2 - 7 } & Coeficientes & Erro Padrão & P & Razão de Chances & LI & LS \\
\hline Faixa Etária & 0,070 & 0,04 & 0,112 & 1,073 & 0,984 & 1,170 \\
BCG & $-0,029$ & 0,19 & 0,880 & 0,972 & 0,669 & 1,411 \\
Parentesco & $-0,081$ & 0,03 & 0,020 & 0,922 & 0,861 & 0,987 \\
Tipo de Convívio & 0,695 & 0,30 & 0,021 & 2,004 & 1,109 & 3,619 \\
Baciloscopia inicial & 0,858 & 0,10 & 0,000 & 2,357 & 1,917 & 2,899 \\
Tempo S/T & 0,762 & 0,07 & 0,000 & 2,142 & 1,850 & 2,481 \\
Localização & $-0,075$ & 0,07 & 0,327 & 0,928 & 0,798 & 1,078 \\
№ de cômodos & $-0,284$ & 0,12 & 0,020 & 0,753 & 0,592 & 0,957 \\
№ de janelas & $-0,427$ & 0,11 & 0,000 & 0,653 & 0,522 & 0,815 \\
\hline
\end{tabular}

Fonte: Protocolo de pesquisa, fevereiro/2015.

Na regressão logística foram utilizadas apenas as variáveis independentes que, através do cálculo do quiquadrado, apresentaram associação estatística significativa.

São elas: faixa etária, vacinação com BCG, parentesco, tipo de convívio, baciloscopia inicial, tempo entre os primeiros sintomas e início do tratamento, localização, número de cômodos do caso índice e número de janelas do caso índice. 
Na Tabela 5 pode-se observar os valores estimados dos coeficientes, o erro-padrão dos coeficientes, assim como seus respectivos níveis descritivos.

\section{DISCUSSÃO}

A Tuberculose é uma das doenças mais antigas e letais da história da humanidade. A descoberta do bacilo de Koch representou grande avanço na luta contra a doença infecciosa, o que significou a redução nas taxas de mortalidade no mundo (SANTIC Z e GALIC K, 2013).

Porém, a atenção aos comunicantes de portadores de Tuberculose ativa ainda não é priorizada, ou seja, a maioria das investigações, resultados e recomendações, envolvendo esse grupo, não são efetivadas na rotina das unidades de saúde, dessa maneira, o número de casos novos da doença ativa, assim como o número de mortes possui uma tendência ao crescimento (LIMA LM et al, 2013).

Trabalhou-se com uma amostra de 1.000 prontuários de comunicantes de portadores de TB ativa, que realizaram a Prova Tuberculínica (PT) no CSE-MARCO entre os anos de 2009 a 2013. Destes, 506 apresentaram PT+ constituindo rendimento de 50,7\%, o que representa uma frequência maior que a prevalência média de infectados pelo M. tuberculosis no Brasil, que é estimada pelo Ministério da Saúde em 30\% (OLIVEIRA MF et al, 2008).

Alguns fatores podem ser determinantes para a frequência de PT+ encontrada nesta investigação, como a demora no diagnóstico de portadores de Tuberculose, em razão de encaminhamentos desnecessários procedentes de outros serviços de saúde que não são capazes de diagnosticar a doença, mas, teriam a obrigação de fazê-lo e as diversas fragilidades do Programa de Controle da Tuberculose (ANDRADE HS et al, 2016).

A organização e operacionalização dos serviços de saúde são os diferenciais responsáveis pelo acesso ao diagnóstico, pelo o tempo recomendado de diagnóstico, além da adesão do tratamento (OLIVEIRA MF et al, 2016; OLIVEIRA MF et al, 2011).

Dentre os usuários que constituíram a amostra, 610 (61,0\%) eram do sexo feminino, e $390(39,0 \%)$ eram do sexo masculino com maior frequência de PT+ entre o sexo masculino $(51,80 \%)$, entretanto, o estudo não constatou associação estatística entre o rendimento da PT. Não foram encontrados estudos que relacionassem a faixa etária como fator determinante para a infecção pelo $M$. tuberculosis, pois a suscetibilidade a doença é universal. Porém, fatores relacionados à competência do sistema imunológico, como nos indivíduos menores de 2 anos e maiores que 60 anos, podem aumentar o risco de adoecimento (BRASIL, 2011b; LAPA e SILVA JR, 2012).

Do total de comunicantes submetidos à Prova Tuberculínica o grupo "Pai/Mãe" apresentou a maior frequência de PT+ com 64,90\%, seguido do grupo "Esposo (a)" com frequência de PT+ de 57,40\%. Indivíduos que convivem com portadores da Tuberculose possuem um risco elevado de infecção e progressão da doença, principalmente, quando esse convívio é intenso e/ou contínuo (LAPA e SILVA JR, 2012; NOGUEIRA JA et al, 2011; KASPROWICZ VO et al, 2011). Constata-se tal afirmativa, visto que o tipo de convívio mais frequente entre comunicantes e portadores de TB ativa foi o contínuo $(n=915)$, além de representar a maior frequência de PT+ $(52,80 \%)$.

Logicamente, que quanto mais confinado for o contato com o portador de Tuberculose ativa, como o fato de dormir diariamente no mesmo cômodo, maiores são as chances de infecção, visto que a concentração de bacilos se torna maior e possibilita uma exposição mais perigosa para o comunicante. Pelo risco apresentado, a investigação e abordagem imediata dos comunicantes são consideradas essenciais para o controle da doença, porém, mesmo sendo um meio efetivo para detectar precocemente novos casos ativos da doença, ainda não há uma sistematização adequada da assistência desses comunicantes (SANTIC Z e GALIC K, 2013; LOPES DMA et al, 2011).

Em relação à vacina $\mathrm{BCG}$, dos pacientes submetidos à Prova Tuberculínica, 154 não eram vacinados, com frequência de $\mathrm{PT}+$ em $63,0 \%$. O grupo de vacinados com 2 doses apresentou frequência de $\mathrm{PT}+$ com $68,20 \%$. A BCG tem sido relacionada a um contexto de resultados falso-positivos, principalmente, quando o tempo entre a vacina e a prova tuberculínica é menor que 3 anos. Nesses casos, a avaliação do comunicante deve considerar os fatores clínico-epidemiológicos, assim como outros fatores de risco e não apenas o resultado isolado da PT (BRASIL, 2011; ZELNER JL et al, 2014).

Além do tempo e tipo de convívio, o exame de baciloscopia no escarro também irá determinar se o caso índice possui maior capacidade de infectar outras pessoas, e, mesmo sendo uma técnica de baixa sensibilidade, ainda é o exame mais utilizado para o diagnóstico da Tuberculose no mundo e possibilita diferenciar a carga bacilar de cada indivíduo doente (PEDRO HSP et al, 2010).

Dentre os indivíduos submetidos a PT, 211 possuíam caso índice com baciloscopia positiva de três cruzes (+++) e apresentaram frequência de positividade de $76,30 \%$. A positividade da baciloscopia tem ligação direta com as chances de infecção. A possibilidade de infecção relaciona-se a carga bacilar de cada doente, pois, quanto maior o número de cruzes na baciloscopia, maior será a carga bacilar liberada no meio de convívio e, 
consequentemente, aumento na possibilidade de infecção (OLIVEIRA MF et al, 2011). Além da carga bacilar, o tempo de exposição também é determinante, principalmente quando o tempo entre o início dos sintomas e início do tratamento do caso índice é prolongado. Dos 1.000 indivíduos submetidos a PT neste estudo, 210 possuíam caso índice que levaram de 04 a 05 meses entre o surgimento dos primeiros sintomas da doença e o início do tratamento, e apresentaram maior frequência de positividade no teste $(73,80 \%)$.

Quanto maior o período sem o tratamento, maiores são as possibilidades de evolução dos sinais e sintomas da doença, dos indivíduos infectados e do risco de morte (LAPA E SILVA JR, 2012).

Em relação às características das residências e moradias observa-se que dos 1.000 comunicantes avaliados, a maior prevalência de PT+ encontrada foi entre os moradores de ruas estreitas (65,10\%). Quando se avaliou o número de cômodos, pode-se observar que 319 comunicantes possuíam como casos índices, moradores de casas com 1 a 3 cômodos, e apresentaram a maior frequência de PT+ (73,04\%). Já em relação ao número de janelas, observa-se que a maior frequência de PT+ $(65,51 \%)$ relacionou-se aos comunicantes que possuíam como casos índices, moradores de casas com 1 a 3 janelas. A Tuberculose continua sendo uma doença de aglomerados humanos, relacionada à pobreza e às condições precárias de moradia que confinam o comunicante e o caso índice em um ambiente reduzido, com pouca ventilação, pouca iluminação e alta carga bacilar exposta (PILLER RVB, 2012).

As condições sociais e econômicas da população são fatores fortemente ligados ao processo saúde-doença, que podem determinar a presença ou não de possíveis infecções e doenças, as maiores taxas de prevalência de doenças infectocontagiosas ainda são mais presentes entre pessoas que residem entre grandes concentrados humanos, que compartilham moradias muitas vezes impróprias (PAULA HC e AGUIAR AC, 2013).

\section{CONCLUSÃO}

A prevalência de Infecção Latente Tuberculosa deste estudo foi de 50,7\%, número que se manteve acima da frequência nacional de $30 \%$ em média. As variáveis significativas para a positividade da Prova Tuberculínica foram: faixa etária do comunicante, presença de fatores de risco, tipo de convívio, ato de dormir no mesmo cômodo, sexo do caso índice, resultado da baciloscopia inicial e tempo entre o início dos sintomas e início do tratamento do caso índice. Com os resultados obtidos no estudo, também foi possível estudar as variáveis envolvidas com o risco de infecção e relacioná-las com o rendimento da prova tuberculínica para avaliar aquelas que possuíam uma relação estatística mais íntima. Dessa forma, visou-se contribuir para incrementar a prevenção da Tuberculose entre os comunicantes de portadores da doença, diminuindo as chances de adoecimentos futuros. $\mathrm{Na}$ análise das variáveis estudadas, observou-se que aquelas que possuíam relação estatística significativa, de acordo com o p-valor e quiquadrado, foram: vacinação com a BCG, faixa etária do comunicante, parentesco entre o comunicante e o caso índice, presença de fatores de risco, tipo de convívio entre o comunicante e o caso índice, ato do comunicante dormir no mesmo cômodo do caso índice, sexo do caso índice, faixa etária do caso índice, baciloscopia inicial do caso índice, tempo entre os primeiros sintomas e início do tratamento do caso índice, localização da residência do caso índice e a quantidade de cômodos e janelas da residência do caso índice.

\section{REFERÊNCIAS}

1. ANDRADE HS, et al. Características clínico-epidemiológicas de casos novos de tuberculose. J Nurs UFPE on line. 2016 julho; 10 (7): 2528-36.

2. BRASIL. Agência Nacional de Saúde Suplementar. Associação Médica Brasileira. Diretrizes Clínicas na Saúde Suplementar. Tuberculose infecção latente: Diagnóstico Rio de Janeiro. ANS Editora. 2011.

3. BRASIL. Ministério da Saúde. Boletim Epidemiológico Tuberculose. Brasília; 2016

4. BRASIL. Ministério da Saúde. Panorama da tuberculose no Brasil. Brasília; 2014.

5. BRASIL. Ministério da Saúde. Secretaria de Vigilância em Saúde. Programa Nacional de Controle da Tuberculose. Manual de Recomendações para o Controle da Tuberculose no Brasil. Brasília; 2011a.

6. BRASIL. Ministério da Saúde. Secretaria de Vigilância em Saúde. Tratamento diretamente observado (TDO) da tuberculose na atenção básica: protocolo de enfermagem. Brasília; 2011b.

7. KASPROWICZ VO, et al. Diagnosing latent tuberculosis in high-risk individuals: rising to the challenge in high burden áreas. $J$ Infect Dis. 2011 Nov; 204(4): S1168-S1178.

8. LAPA E SILVA JR. Novos Aspectos da Patogenia da Tuberculose. Pulmão RJ. 2012; 21 (1): 10-14.

9. LIMA LM, et al. O programa de controle da tuberculose em Pelotas/RS, Brasil: investigação de contatos intradomiciliares. Rev. Gaúcha Enferm. 2013 junho; 34(2): 102-110.

10. LOPES DMA, et al. Diagnóstico e tratamento da tuberculose latente em pacientes com doenças inflamatórias crônicas e uso de imunobiológicos inibidores do TNF-a. J. bras. pneumol. 2011 junho; 37(3): 308-316.

11. NOGUEIRA JA, et al. Enfoque familiar e orientação para a comunidade no controle da tuberculose. Rev. bras. epidemiol. 2011 junho; 14(2): 207-216.

12. OLIVEIRA MF, et al. A porta de entrada para o diagnóstico da tuberculose no sistema de saúde de Ribeirão Preto/SP. Rev. esc. enferm. USP. 2011 Ago; 45(4): 898-904.

13. OLIVEIRA SMVL, et al. Efeito booster na prova tuberculínica em um hospital universitário de Mato Grosso do Sul. Rev. bras. saúde ocup. 2008 junho; 33 (117): 72-76.

14. OLIVEIRA SMVL, et al. Teste tuberculínico: pesquisa operacional no Mato Grosso do Sul. J. bras. pneumol. 2011 Out; $37(5): 646-654$.

15. PAULA HC, Aguiar AC. Abandono do tratamento da tuberculose na estratégia saúde da família: estudo qualitativo em uma área programática do Rio de Janeiro. Revista Baiana de Saúde Pública. 2013 Jan-março; 37(1): 192-204.

16. PEDRO HSP, et al. Baciloscopia para Tuberculose pulmonar. Estudo multicêntrico do esfregaço para baciloscopia de escarro no diagnóstico da tuberculose pulmonar segundo a Organização Mundial da Saúde e o Ministério da Saúde. Revista de Patologia Tropical. 2010 Out-Dez; Vol. 39 (4): 273-282.

17. PILLER RVB. Epidemiologia da tuberculose. Pulmão, Rio de Janeiro. 2012; 21(1): 4-9.

REAS/EJCH | Vol.Sup.30 | e851 | DOI: https://doi.org/10.25248/reas.e851.2019 Página 7 de 8 
18. SANTIC Z, Galic K. Epidemiology of tuberculosis during the period 1703-2011: honoring the world tuberculosis day. Mater Sociomed, 2013; 25(4)

19. ZELNER JL, et al. Bacillus Calmette-Guérin and Isoniazid Preventive Therapy Protect Contacts of Patients with Tuberculosis. Am J Respir Crit Care Med. 2014 abril; 189 (7): 853-859. 